\title{
Tight-Binding Model for Spontaneous Magnetism of Quantum Dot Lattices
}

\author{
P. Koskinen ${ }^{1}$, L. Sapienza ${ }^{2}$ and M. Manninen ${ }^{1}$ \\ ${ }^{1}$ Department of Physics, FIN-40014 University of Jyväskylä, Finland \\ ${ }^{2}$ Universita degli studí di Padova, 35100 Padova, Italy
}

Received December 17, 2002; accepted March 7, 2003

PACS Ref: 73.21.La, 75.10.Lp, 75.75.+a

\begin{abstract}
We use a simple tight-binding model to study the magnetism of twodimensional quantum dot lattices with 1 to 12 electrons per dot. The results show that in the middle of an electron shell the lattice favours antiferromagnetism while with nearly empty or full shells ferromagnetism is favoured. The size of the antiferromagnetic region increases with the coordination number of the dot. A one-dimensional dot lattice shows a spin-Peierls transition. The results for a square lattice are in good agreement with density functional calculations of Koskinen et al. [13].
\end{abstract}

\section{Introduction}

During the last decades low-dimensional semiconductor heterostructures have been a rich source of discoveries. Analogies of quantum dots with real atoms have been confirmed by observation of the shell structure and magnetism due to Hund's first rule [1-4]. Contrary to real atoms or ions where the confining potential is fixed, in quantum dots, or artificial atoms, the shape of the confining potential as well as the electron number can be designed at will (for reviews, see for example, Refs. [5,6]). Moreover, in making lattices of quantum dots one is not restricted to the energetically most favourable structure as in the case of real atoms, but the lattice can have the desired symmetry. This opens up possibilities of magnetic structures that can not be observed with natural atoms.

In elemental solids, magnetism is observed only in the cases where the magnetic moment of the atoms is determined by the inner $\mathrm{d}$-shell or f-shell. If the magnetic moment of an open shell atom is determined by the sp-shell like in sodium or boron, the magnetism disappears in the solid phase due to metallic or covalent bonding which favour nonmagnetic ground states. The situation could be different if the lattice constant and structure could be chosen at will. At large enough lattice constant the solid would change from a nearly free electron metal to a tightbinding system with very narrow bands. In this case, for example sodium would resemble a Hubbard system where the ground state is expected to be an antiferromagnet $[7,8]$.

In quantum dot lattices the structure and lattice constant (as well as the internal properties of the quantum dot) can be nearly freely chosen. It is then possible to obtain magnetism caused by the outer electronic shell. Most of the studies of two-dimensional dot lattices relate to the simple Hubbard model or its extensions [8-12]. In this case one is interested in the region where each dot has less than two electrons. In the simplest case there is only one band. When it is half full the system is antiferromagnetic, irrespective of the lattice structure. If the band is less (or more) than half full the situation gets much more subtle. There are general theories that in the limit of weak coupling between the dots (the ratio of the Hubbard parameters, $t / U$, is small) the system becomes ferromagnetic even if the departure from the half-filling is infinitesimally small $[9,8]$. Similarly, when the filling of the band is small the system becomes paramagnetic. When the hopping parameter increases to the region $t / U \sim 1$ the situation becomes less clear.

Increasing the number of electrons per $\operatorname{dot}, N$, makes the magnetic coupling between the dots more complicated and the Hubbard-type models necessarily need more parameters. Another approach to the electronic structure of the quantum dot lattice is the density functional approach. Koskinen et al. [13] have recently calculated the magnetic structure of square lattices of quantum dots containing from 1 to 12 electrons per dot. Their result shows that when the lattice constant is large enough the dot lattice shows magnetic order, which in general is antiferromagnetic for a half full electron shell and otherwise ferromagnetic (full-shell dots naturally form a nonmagnetic lattice).

The purpose of this paper is to demonstrate that the systematics of the results of Koskinen et al. [13] can be understood with the simplest possible tight-binding model with rigid energy bands. Furthermore, we use the model to predict, for example, that the size of the antiferromagnetic phase increases with the coordination number, i.e., the more frustrated the system is the more antiferromagnetism is favoured.

The structure of the paper is as follows. First we will give the theoretical model in Section 2. In Section 3 we present results for a square lattice and in Section 4 we discuss other 2D-lattices. The long-wave-length antiferromagnetism and spin-Peierls transition is discussed in Section 5. A short conclusion is given in Section 6.

\section{Tight-binding model}

We assume a periodic two-dimensional lattice of identical quantum dots. Each individual dot has localized eigenstates forming a shell structure related to that of a $2 \mathrm{D}$ harmonic oscillator. The three lowest electron shells are filled with $1 \mathrm{~s}, 1 \mathrm{p}$, and $2 \mathrm{~s}$ and $1 \mathrm{~d}$ electrons, respectively. Due to the fact that the effective potential is usually not exactly harmonic the $2 \mathrm{~s}$ and $1 \mathrm{~d}$ electron levels of the third shell are not exactly degenerate but separated by a small energy difference $\Delta_{\mathrm{sd}}$. If the last occupied electron shell is partially filled the electrons obey the first Hund's rule and maximize the spin. The related exchange splitting lifts the degeneracy 


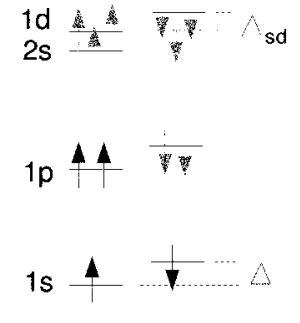

(a)

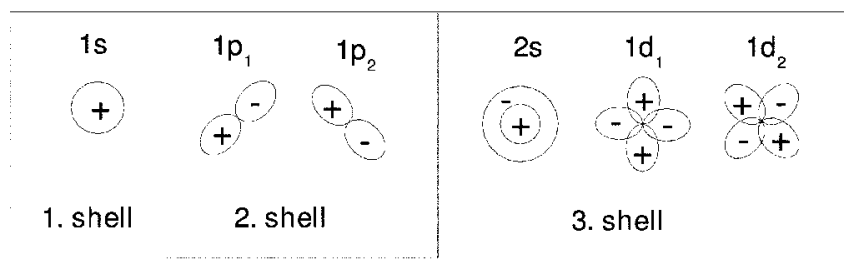

(c)

Fig. 1. (a) A schematic picture of the electronic structure of an individual quantum dot with 4 electrons. The black arrows indicate the filled states and the grey arrows the empty levels. $\Delta$ is the spin splitting assumed to be the same for all shells and $\Delta_{\text {sd }}$ the energy difference between the $2 \mathrm{~s}$ and $1 \mathrm{~d}$ levels. (b) The antiferromagnetic square lattice with the unit shell (dashed line) and possible nearest neighbour hoppings (solid lines). (c) Symmetries of the energy levels of the three shells considered.

of the spin-up and spin-down levels. We denote this energy difference by $\Delta$, as illustrated in Fig. 1(a). We assume a quantum dot where the energy difference between different shells is much larger than the exchange splitting $\Delta$. With this approximation we can consider (in the tight-binding model) only the open shell in question and neglect the full "core" levels as well as the empty shells. A schematic picture of the electronic structure of an isolated quantum dot is shown in Fig. 1(a).

In a square lattice we have to take at least two dots per unit cell in order to describe the antiferromagnetic order. We denote these dots with $a$ and $b$, as shown in Fig. 1(b). The figure also shows the possible hoppings of electrons from one dot to its neighbours. Note that in a square lattice the nearest neighbour hopping is only possible from $a$ to $b$ and vice versa. The simple tight-binding Hamiltonian can now be written for a given spin (up or down) as

$$
\begin{aligned}
H= & \sum_{i} \sum_{k=1}^{m}\left(\varepsilon_{a k} a_{i}^{\dagger k} a_{i}^{k}+\varepsilon_{b k} b_{i}^{\dagger k} b_{i}^{k}\right) \\
& +\sum_{i, j} \sum_{k, l=1}^{m}\left(t_{i j}^{k l} a_{i}^{\dagger k} b_{j}^{l}+t_{i j}^{k l} b_{i}^{\dagger k} a_{j}^{l}+t_{i j}^{k l} a_{i}^{\dagger k} a_{j}^{l}+t_{i j}^{k l} b_{i}^{\dagger k} b_{j}^{l}\right),
\end{aligned}
$$

where $i$ and $j$ run over the unit cells and $k$ and $l$ over the $m$ electron states in a dot. $\varepsilon_{a i}$ and $\varepsilon_{b i}$ are the energy levels in dots $a$ and $b$, respectively. $t_{i j}^{k l}$ is the hopping parameter between the electron state $k$ in site $i$ and state $l$ in cell $j$. The operator $a_{i}^{l},\left(b_{i}^{l}\right)$ is the usual fermionic annihilation (H.c. for creation) operator for a single-particle state $\left|a_{i}^{k}\right\rangle\left(\left|b_{i}^{k}\right\rangle\right)$ in a unit cell $i$, dot state $k=\left(1 \mathrm{~s}, 1 \mathrm{p}_{1}, \ldots\right)$ and $\operatorname{dot} a(b)$. The eigenstates of the Hamiltonian can easily be obtained by the ansatz $|\psi\rangle=\sum_{i} \sum_{k} \exp \left(\mathrm{i} \boldsymbol{q} \cdot \boldsymbol{r}_{i}\right)\left(\alpha_{k}\left|a_{i}^{k}\right\rangle+\beta_{k}\left|b_{i}^{k}\right\rangle\right)$, yielding ultimately the whole band structure. The lattice symmetry is fixed by choosing the suitable non-zero hopping parameters $t_{i j}^{k l}$ (which can also depend on $a$ and $b$ ).
We considered only nearest neighbour tunnelling and ignored hoppings between different energy shells. This means that when filling the dots with electrons, we can consider only one shell at a time, i.e. "the valence shell", and assume that the "core" does not play a dynamic role any more, just as in the case of real atoms. With this justification, we can solve the band structure one shell at a time. For example, if we have slightly more than two electrons per dot, so that we are filling the $1 \mathrm{p}$-states, the energy bands arise only from the $\mathrm{p}_{1}$ and $\mathrm{p}_{2}$ states as schematically shown in Fig. 1(c).

In the antiferromagnetic case we assume a spin splitting $\Delta$ to exist between neighbouring atoms. If an electron with spin up is located at the $\operatorname{dot} a$, all the dots $b$ assume a higher energy for spin-up state. Correspondingly, the spindown states in $b$-dots have the same energy as the spin-up states in dots $a$. In the ferromagnetic case the spin-up (majority spin) energies are the same for all the dots, whereas for the minority spin the energy is higher by $\Delta$. Thus in antiferromagnetic case the bands are identical for both spins, whereas in ferromagnetic case the spin-downbands are lifted by $\Delta$. We will use $\Delta$ as the unit of energy in our model. In reality, the spin splitting will depend on the number of electrons in the dot and on the interdot distance. However, since we look at the magnetic properties as a function of the hopping parameters $t$, the relevant parameter determining the qualitative features is in any case $t / \Delta$ or its inverse.

Figure 1(c) shows schematically the single particle orbitals of individual dots. The first energy shell has only one state and one $t$-parameter. The second shell has two orthogonal p-states. Due to the symmetry of the square lattice hopping is forbidden from $1 \mathrm{p}_{1}$ to $1 \mathrm{p}_{2}$. Within this shell we have then two nonzero hopping parameters, $t^{\mathrm{p}_{1} \mathrm{p}_{1}}$ and $t^{\mathrm{p}_{2} \mathrm{p}_{2}}$. In the third shell the possible nonzero hopping integrals are $t^{\mathrm{s}_{2} \mathrm{~s}_{2}}, t^{\mathrm{d}_{1} \mathrm{~d}_{1}}, t^{\mathrm{d}_{2} \mathrm{~d}_{2}}$, and $t^{\mathrm{s}_{2} \mathrm{~d}_{2}}$.

The band structure for a given energy shell is obtained by solving the Hamiltonian of Eq. (1). Since we fix $\Delta$ to be independent of the number of electrons the model is a "fixed band model" [14], i.e., the bands do not depend on the number of electrons. After the calculation of the band structure for the antiferromagnetic (AF) and ferromagnetic (F) cases, we determine the total energies by integrating the band energies over the occupied parts of the bands. For a given electron number $N$ the magnetism of the ground state is simply determined by which structure gives the lower total energy. Note that in our model the open shell structures are always either ferro- or antiferromagnetic. A nonmagnetic solution is energetically not favourable since we postulate that there is an exchange splitting $\Delta$.

\section{Results for a square lattice}

The main results for a square lattice are shown in Fig. 2, where we show the "phase diagram" of the quantum dot lattice as a function of $t / \Delta$ and the number of electrons per dot. In this case we have, for simplicity, chosen all nonzero $t$-parameters to have the same value. The ground state is ferromagnetic, except close to the middle of any electron shell $(N=1,4,9)$. When $t$ goes to zero (corresponding to the large lattice constant limit in a real lattice), the width of the antiferromagnetic area goes to zero. The small 


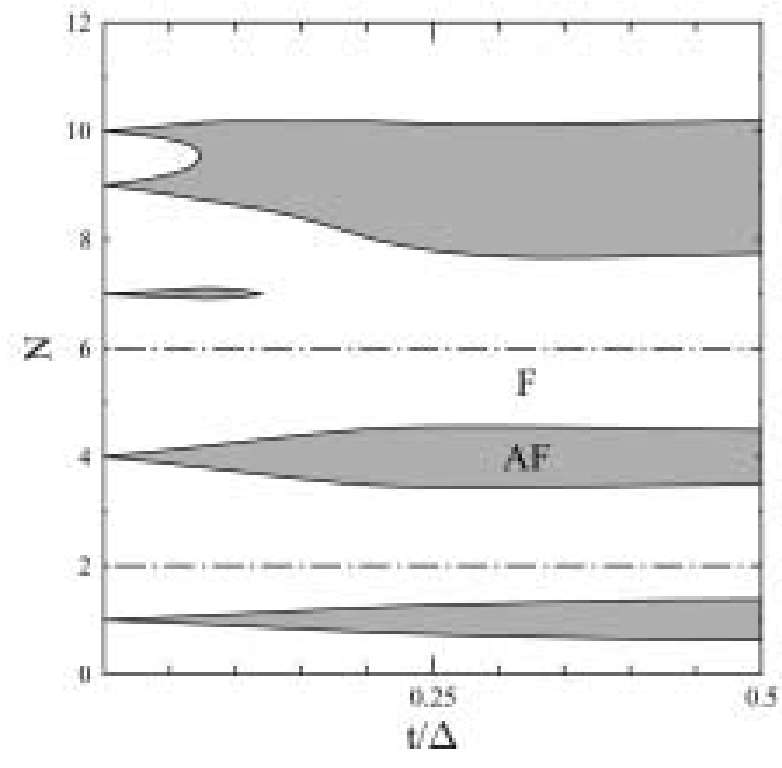

Fig. 2. Magnetic phase diagram for a square lattice showing antiferromagnetic (AF, grey) and ferromagnetic (F, white) regions. $N$ is the number of atoms per dot and $t / \Delta$ is the ratio of the hopping parameter and the exchange splitting. $\Delta_{\mathrm{sd}} / \Delta=1 / 2$. The hopping parameter is assumed to be the same between all states ( $t=0$ if symmetry prevents the hopping).

antiferromagnetic area at $N=7$ is caused by half-filling of the subband of 2 s-electrons which are separated by $\Delta_{\mathrm{sd}}$ from the d-electrons $\left(\Delta_{\mathrm{sd}}=\Delta / 2\right.$ in Fig. 2). In this case the total spin of an individual dot is $S=1 / 2$. Similarly the antiferromagnetism at $N=10$ (in the small $t$ limit) is caused by a full $2 \mathrm{~s}$ band separated from the half-full $\mathrm{d}-$ band. In this case the total spin of a single dot is $S=1$. For $N=9$ the antiferromagnetism corresponds to the total polarization of the sd-shell, with total spin of a single dot being $S=3 / 2$.

An increase of $t / \Delta$ eventually makes the narrow bands of the of separate dot levels to merge to a wide antiferromagnetic region centered at half-full sd-shell. Interestingly, the $N=7$ case changes from antiferromagnetism to ferromagnetism when $t / \Delta$ ingreases. The opposite happens for $N=8$. Qualitatively the magnetic phase diagram of the sd-band is insensitive to the ratio $\Delta_{\text {sd }} / \Delta$ as shown in Fig. 3. In all cases shown the electron numbers $N=7,9$ or 10 show antiferromagnetism at the limit of small $t / \Delta$ and the antiferromagnetic region at large $t / \Delta$ approaches to the same width. In the intermediate values of $t / \Delta$ the details are different.

In the above results we assumed that all the $t$-parameters have the same value. We have also studied the effect of changing the ratios of different $t$-parameters as well as the signs of them. The general conclusion is that while the detailed positions of the phase boundaries change slightly, the overall phase diagram remains the same. The ratio $\Delta_{\text {sd }} / \Delta$ has a much larger effect (shown in Fig. 3) to the phase diagram than the ratios of different $t$-parameters. The reason that the sign of $t$ does not affect the results much is due to the fact that the rigid density of states of our model is nearly symmetric with respect of the band center, irrespective of the ratios of the different $t$-parameters.

Recently, Koskinen et al. [13] have studied magnetism of dot lattices using density functional theory with the local spin density approximation. They have computed the ground state of square lattices with 1 to 12 electrons as a

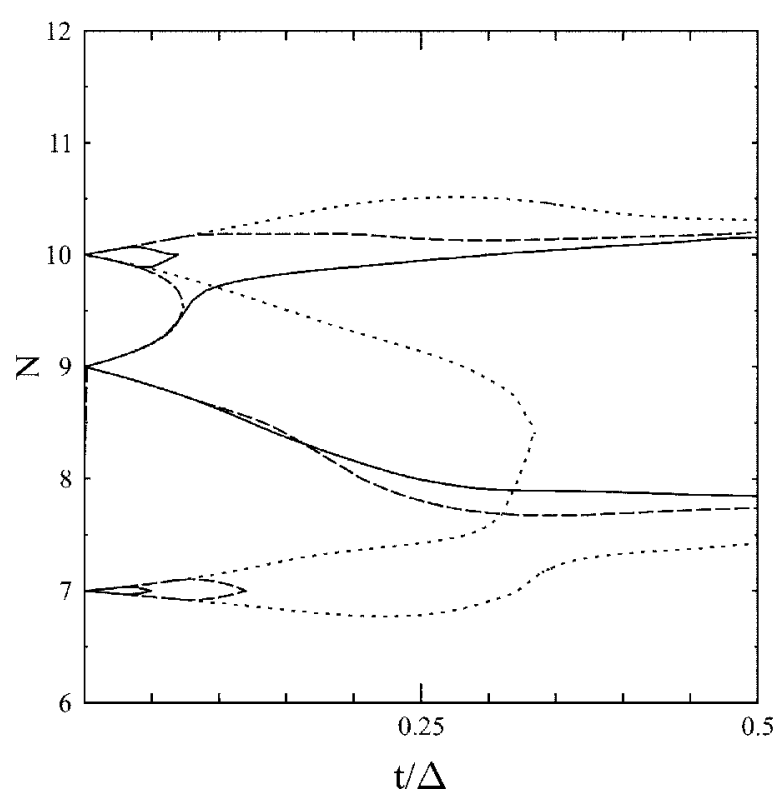

Fig. 3. Dependence of magnetism of a square lattice on the energy difference between the $2 \mathrm{~s}$ and $1 \mathrm{~d}$ levels. The different lines show the boundaries between the antiferromagnetic and ferromagnetic regions. Solid line: $\Delta_{\mathrm{sd}} / \Delta=1 / 4$, dashed line $\Delta_{\mathrm{sd}} / \Delta=1 / 2$ (same as in Fig. 2), dotted line $\Delta_{\mathrm{sd}} / \Delta=1$.

function of the lattice constant. The results of our simple tight-binding model are in excellent agreement with the magnetic structures obtained by Koskinen et al. for large values of the lattice constant. At small values of lattice constant, corresponding to large values of $t / \Delta$ they obtained nonmagnetic ground states. This is not possible in our model since we assume a fixed nonzero exchange splitting. Koskinen et al. only considered integer number of electrons per dot and found that filled and half-filled shells are insulators while the ferromagnetic states are metallic (no band-gap). If the occupation number is not an integer number, even the antiferromagnetic phase is always metallic. Note especially, that strictly speaking the closed shells (and other integer occupation numbers) are only singular points when increasing the occupation number.

Hartree-Fock results for the Hubbard model [10,14] for the 1 s-band, i.e., for $N=0, \ldots, 2$, result in a phase diagram very similar to ours. The main difference being a paramagnetic phase at large values of $t / \Delta$ and at nearly empty or full 1s-band. The Hartree-Fock approximation takes the electron-electron interaction into account in determining the exchange splitting while we assume it to be fixed.

\section{Antiferromagnetism in other lattices}

Using the simple tight-binding model it is easy to calculate the phase diagrams also for other lattices. Figure 4 shows phase diagram for $1 \mathrm{~s}$ and $1 \mathrm{p}$ shells for different twodimensional lattices: square, honeycomb and hexagonal. Again we assume the $t$-parameters between all possible transitions between the p-electrons to be the same (note that in principle the $t$-parameters for p-electrons are angledependent). The mid-shell antiferromagnetic area is narrower in the case of the square lattice than in the case of the hexagonal lattice. It actually seems quite counter-intuitive that a frustrated system like a hexagonal lattice would 


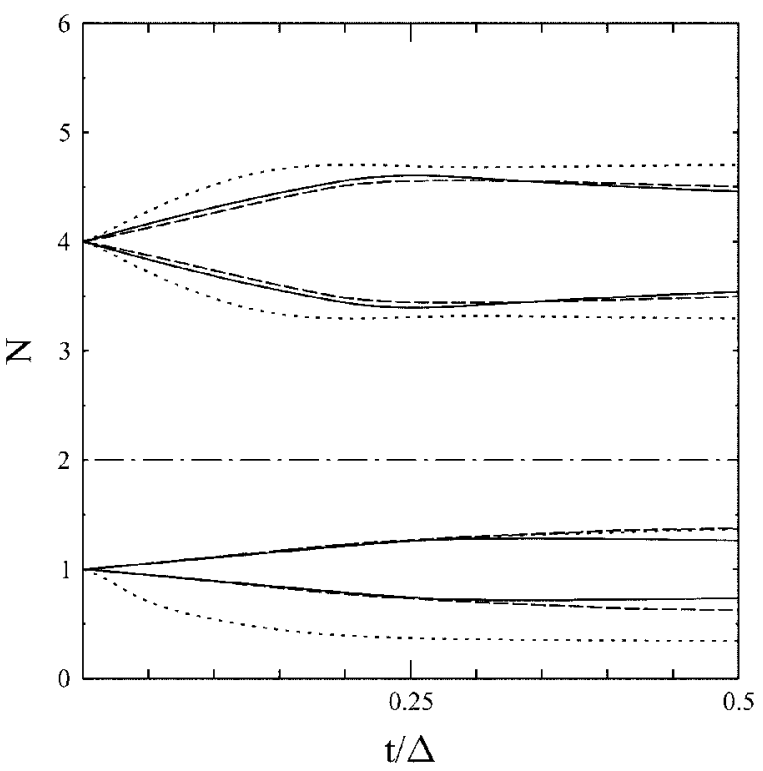

Fig. 4. Phase diagram for honeycomb (solid line), square (dashed line) and hexagonal (dotted line) lattice. The lines separate the antiferromagnetic and ferromagnetic regions. Note that the antiferromagnetic region is larger for frustrated hexagonal lattice than for the square lattice.

exhibit antiferromagnetism to the largest extent. A closer examination reveals that a more important ingredient to the antiferromagnetic behaviour is the coordination number $c$ of the lattice. The structure of the 1s-band can be easily derived analytically. For example, for a $D$-dimensional cubic lattice we get

$\varepsilon(q)=\frac{1}{2}\left(\varepsilon_{a}+\varepsilon_{b}\right) \pm \frac{1}{2} \sqrt{\left(\varepsilon_{a}-\varepsilon_{b}\right)^{2}+4 t^{2}|A(\boldsymbol{q})|^{2}}$,

where $A(q)=\sum_{i}^{n . n \cdot} \mathrm{e}^{-\mathrm{i} \boldsymbol{q} \cdot \boldsymbol{r}_{i}}$ describes all the possible nearestneighbour hoppings. The essential point here is, that with zero or small wave-vector (or band extrema in general) $|A(\boldsymbol{q})| \approx c$. Expanding (2) for small $t$, we get for the lowest bands

$$
\begin{array}{ll}
\varepsilon(0)=-\frac{\Delta}{2}-\frac{1}{\Delta}(|A| t)^{2}, & \text { for antiferromagnetic case } \\
\varepsilon(0)=-\frac{\Delta}{2}-|A| t, & \text { for ferromagnetic case. }
\end{array}
$$

The lowest-lying states for the antiferromagnetic case are lower in energy than those for the ferromagnetic case if $c t>\Delta$. For a fixed $t$ the electron number $N$, where the system becomes antiferromagnetic, is the smaller the larger is the coordination number. This explains the wider antiferromagnetic regions for lattices with larger $c$ (for a simple cubic $c=2^{D}$ ). It may be mentioned that in an infinite-dimensional lattice the system would be antiferromagnetic for any number of electrons.

\section{Spin-Peierls transition in one-dimensional quatum dot lattice}

In the previous sections we have studied the antiferromagnetic phase only in its simplest form where the neighbouring dots have opposite spins (in the hexagonal lattice two of the neighbours have parallel spins). In

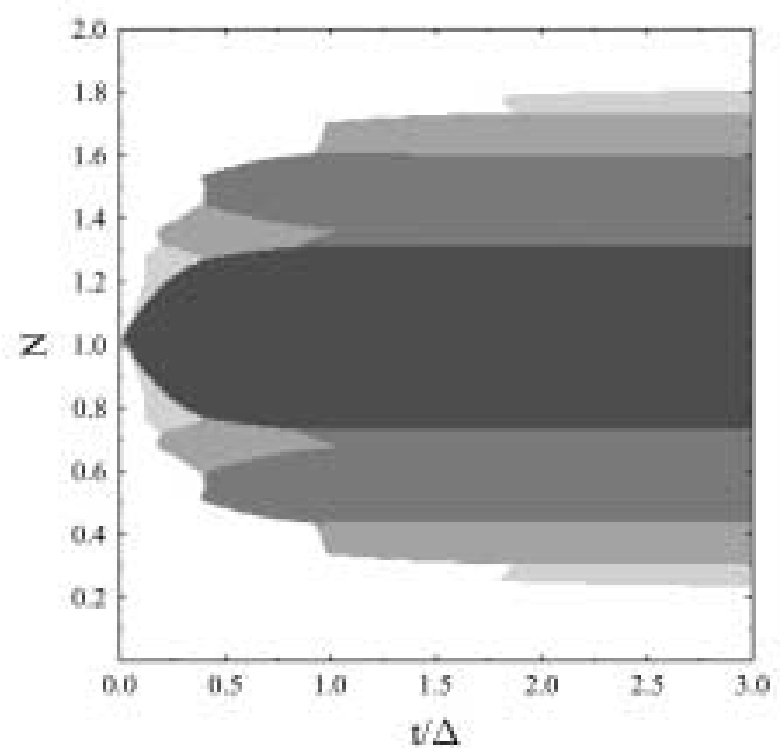

Fig. 5. Spin-Peierls transition in one-dimensional dot lattice. $N$ is the number of electron in the dot and $t / \Delta$ is the ratio between the hopping parameter and the exchange splitting. The grey areas from dark to light

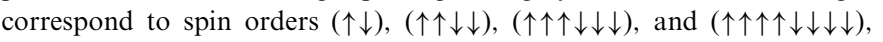
respectively.

reality the "spin-wave" of the antiferromagnetic phase can have much longer wavelength. We will study this possibility only in the case of the one-dimensional lattice which is expected to exhibit a spin-Peierls transition. To this end we calculated the band structures and the tight binding total energies for one-dimensional dot lattices with 2, 4, 6, and 8 dots in the unit cell and the spin

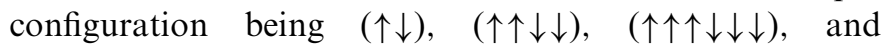

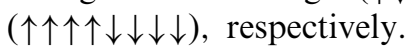

Figure 5 shows the ground magnetic structure for a onedimensional dot lattice as a function of $t / \Delta$ and $N$. The spin-Peierls transition is clearly seen for large values of $t / \Delta$ where strips corresponding to different spin density waves appear. The energetically most favourable occupation number for a given wavelength of the spin density wave is $N=2 / n$, where $n$ is the number of dots in the unit cell (wavelength). It is interesting to note that for small $t / \Delta$ also small regions of long wavelength antiferromagnetism appear.

In the limit of small $t / \Delta$ antiferromagnetic order exists only for the half-filled band, with a wavelength of two unit cells. When $t / \Delta$ increases antiferromagnetism with longer wavelength starts to exist, according to the spin-Peierls transition. The longer the wavelength the larger $t / \Delta$ has to be in order for the spin-Peierls transition to win the ferromagnetism.

\section{Conclusions}

We have investigated the spontaneous magnetism of quantum dot lattices with up to 12 electrons per dot using a tight-binding approach with rigid energy bands. This, perhaps the simplest possible model, shows qualitatively the same results as the previously obtained densityfunctional calculations [13], when the lattice constant is sufficiently large. Lattices made of dots with half-filled shells are antiferromagnetic insulators and those made of dots with filled shells are non-magnetic insulators. Close to 
the half-filling of each shell the dot lattice is a metallic antiferromagnet. When the electron shell is only slightly filled or almost full ferromagnetic order is obtained. These general results are not sensitive to the parameters of the tight-binding model.

The region of antiferromagnetism increases with the coordination number of the dots. This means that even the frustrated hexagonal lattice has a larger region of ferromagnetism than the unfrustrated square lattice.

The one-dimensional lattice shows spin-Peierls transitions at sufficiently large values of the hopping parameters.

\section{Acknowledgements}

We would like to thank Matti Koskinen and Stephanie Reimann for several useful discussions. This work has been supported by the Academy of Finland under the Finnish Centre of Excellence Programme 2000-2005). (Project No. 44875, Nuclear and Condensed Matter Programme at JYFL). One of us (PK) is grateful for the Väisälä foundation for financial support.

\section{References}

1. Tarucha, S., Austing, D.G., van der Haage, R.J. and Kouwenhoven, L., Phys. Rev. Lett. 77, 3613 (1996).

2. Koskinen, M., Reimann, S. M. and Manninen, M., Phys. Rev. Lett. 79, 1389 (1997).

3. Hirose, K. and Wingreen, N., Phys. Rev. B 59, 4604 (1999).

4. Yannouleas, C. and Landman, U., Phys. Rev. Lett. 82, 5325 (1999).

5. Chakraborty, T., "Quantum Dots: A Survey of the Properties of Artificial Atoms," (North-Holland, Amsterdam, 1999).

6. Reimann, S. M. and Manninen, M., Rev. Mod. Phys. 74, 1283 (2002).

7. Vollhardt, D. in "Perspectives in Many-Body Physics," (ed. by R. A. Broglia, J. R. Schrieffer, and P. F. Bortignon), (North-Holland, Amsterdam, 1994).

8. Tasaki, H., Prog. Theor. Phys. 99, 489 (1998).

9. Nagaoka, Y., Phys. Rev. 147, 392 (1966).

10. Hirsch, J. E., Phys. Rev. B 31, 4403 (1985).

11. Freericks, J. K. and Jarrel, M., Phys. Rev. Lett. 74, 186 (1995).

12. Zanchi, D. and Schulz, H. J., Phys. Rev. B 61, 13609 (2000).

13. Koskinen, M., Reimann, S. M. and Manninen, M., Phys. Rev. Lett. 90, $066802(2003)$

14. Mattis, D. C., "Theory of Magnetism I," (Springer, Heidelberg, 1988). 